Web-based Supplementary Material for Manuscript

\title{
Blinded sample size recalculation in clinical trials with binary composite endpoints
}

Anja Sander, Geraldine Rauch and Meinhard Kieser 


\section{Description of considered scenarios}

Table 1: Considered scenario 1: $\Delta_{C E}=0.06, p_{M C}=0.0125, \Delta_{M C}=0.003, \alpha_{M C}=0.0125$.

\begin{tabular}{cccrrrrr}
\hline$p_{C E}^{C}$ & $p_{C E}^{I}$ & $p_{C E}$ & Corr $_{H_{0}}\left(T_{C E}, T_{M C}\right)$ & Corr $_{H_{1}}\left(T_{C E}, T_{M C}\right)$ & $\alpha_{C E}$ & $n_{0}$ & $\mathbb{E}\left(\right.$ Corr $\left._{\text {blind }}\right)$ \\
\hline 0.09 & 0.03 & 0.06 & 0.379 & 0.447 & 0.01368 & 291 & 0.445 \\
0.10 & 0.04 & 0.07 & 0.357 & 0.411 & 0.01358 & 337 & 0.410 \\
0.11 & 0.05 & 0.08 & 0.339 & 0.382 & 0.01349 & 381 & 0.382 \\
0.12 & 0.06 & 0.09 & 0.323 & 0.358 & 0.01342 & 424 & 0.358 \\
0.13 & 0.07 & 0.10 & 0.308 & 0.338 & 0.01336 & 466 & 0.338 \\
0.14 & 0.08 & 0.11 & 0.295 & 0.01331 & 507 & 0.320 \\
0.15 & 0.09 & 0.12 & 0.284 & 0.305 & 0.01327 & 547 & 0.305 \\
0.16 & 0.10 & 0.13 & 0.273 & 0.291 & 0.01323 & 586 & 0.291 \\
0.17 & 0.11 & 0.14 & 0.263 & 0.268 & 0.01320 & 624 & 0.279 \\
0.18 & 0.12 & 0.15 & 0.254 & 0.258 & 0.01314 & 660 & 0.268 \\
0.19 & 0.13 & 0.16 & 0.246 & 0.248 & 0.01312 & 731 & 0.258 \\
0.20 & 0.14 & 0.17 & 0.238 & 0.240 & 0.01310 & 764 & 0.249 \\
0.21 & 0.15 & 0.18 & 0.231 & 0.232 & 0.01308 & 797 & 0.240 \\
0.22 & 0.16 & 0.19 & 0.224 & 0.225 & 0.01306 & 828 & 0.232 \\
0.23 & 0.17 & 0.20 & 0.218 & 0.01304 & 858 & 0.218 \\
0.24 & 0.18 & 0.21 & 0.212 & 0.211 & 0.01303 & 888 & 0.212 \\
0.25 & 0.19 & 0.22 & 0.206 & & & \\
\hline
\end{tabular}

Table 2: Considered scenario 2: $\Delta_{C E}=0.1, p_{M C}=0.075, \Delta_{M C}=0.05, \alpha_{M C}=0.0125$.

\begin{tabular}{cccrrrrr}
\hline$p_{C E}^{C}$ & $p_{C E}^{I}$ & $p_{C E}$ & Corr $_{H_{0}}\left(T_{C E}, T_{M C}\right)$ & Corr $_{H_{1}}\left(T_{C E}, T_{M C}\right)$ & $\alpha_{C E}$ & $n_{0}$ & $\mathbb{E}\left(\right.$ Corr $\left.^{\text {blind }}\right)$ \\
\hline 0.15 & 0.05 & 0.10 & 0.793 & 0.854 & 0.01843 & 155 & 0.854 \\
0.20 & 0.10 & 0.15 & 0.667 & 0.674 & 0.01619 & 219 & 0.678 \\
0.25 & 0.15 & 0.20 & 0.577 & 0.565 & 0.01513 & 262 & 0.569 \\
0.30 & 0.20 & 0.25 & 0.509 & 0.488 & 0.01452 & 290 & 0.493 \\
0.35 & 0.25 & 0.30 & 0.454 & 0.429 & 0.01412 & 308 & 0.435 \\
0.40 & 0.30 & 0.35 & 0.408 & 0.382 & 0.01384 & 319 & 0.388 \\
0.45 & 0.35 & 0.40 & 0.369 & 0.342 & 0.01363 & 325 & 0.349 \\
0.50 & 0.40 & 0.45 & 0.333 & 0.308 & 0.01347 & 327 & 0.315 \\
0.55 & 0.45 & 0.50 & 0.302 & 0.278 & 0.01334 & 326 & 0.285 \\
\hline
\end{tabular}


Table 3: Considered scenario 3: $\Delta_{C E}=0.18, p_{M C}=0.08, \Delta_{M C}=0.06, \alpha_{M C}=0.0125$.

\begin{tabular}{rrrrrrrr}
\hline$p_{C E}^{C}$ & $p_{C E}^{I}$ & $p_{C E}$ & $\operatorname{Corr}_{H_{0}}\left(T_{C E}, T_{M C}\right)$ & Corr $_{H_{1}}\left(T_{C E}, T_{M C}\right)$ & $\alpha_{C E}$ & $n_{0}$ & $\mathbb{E}\left(\right.$ Corr $\left.^{\text {blind }}\right)$ \\
\hline 0.24 & 0.06 & 0.15 & 0.626 & 0.701 & 0.01566 & 71 & 0.702 \\
0.29 & 0.11 & 0.20 & 0.550 & 0.583 & 0.01487 & 89 & 0.590 \\
0.34 & 0.16 & 0.25 & 0.490 & 0.502 & 0.01437 & 103 & 0.511 \\
0.39 & 0.21 & 0.30 & 0.440 & 0.440 & 0.01403 & 114 & 0.450 \\
0.44 & 0.26 & 0.35 & 0.397 & 0.390 & 0.01378 & 122 & 0.402 \\
0.49 & 0.31 & 0.40 & 0.359 & 0.349 & 0.01358 & 127 & 0.361 \\
0.54 & 0.36 & 0.45 & 0.324 & 0.313 & 0.01343 & 129 & 0.326 \\
0.59 & 0.41 & 0.50 & 0.293 & 0.281 & 0.01331 & 130 & 0.295 \\
\hline
\end{tabular}

2. Results for the unrestricted recalculation approach 

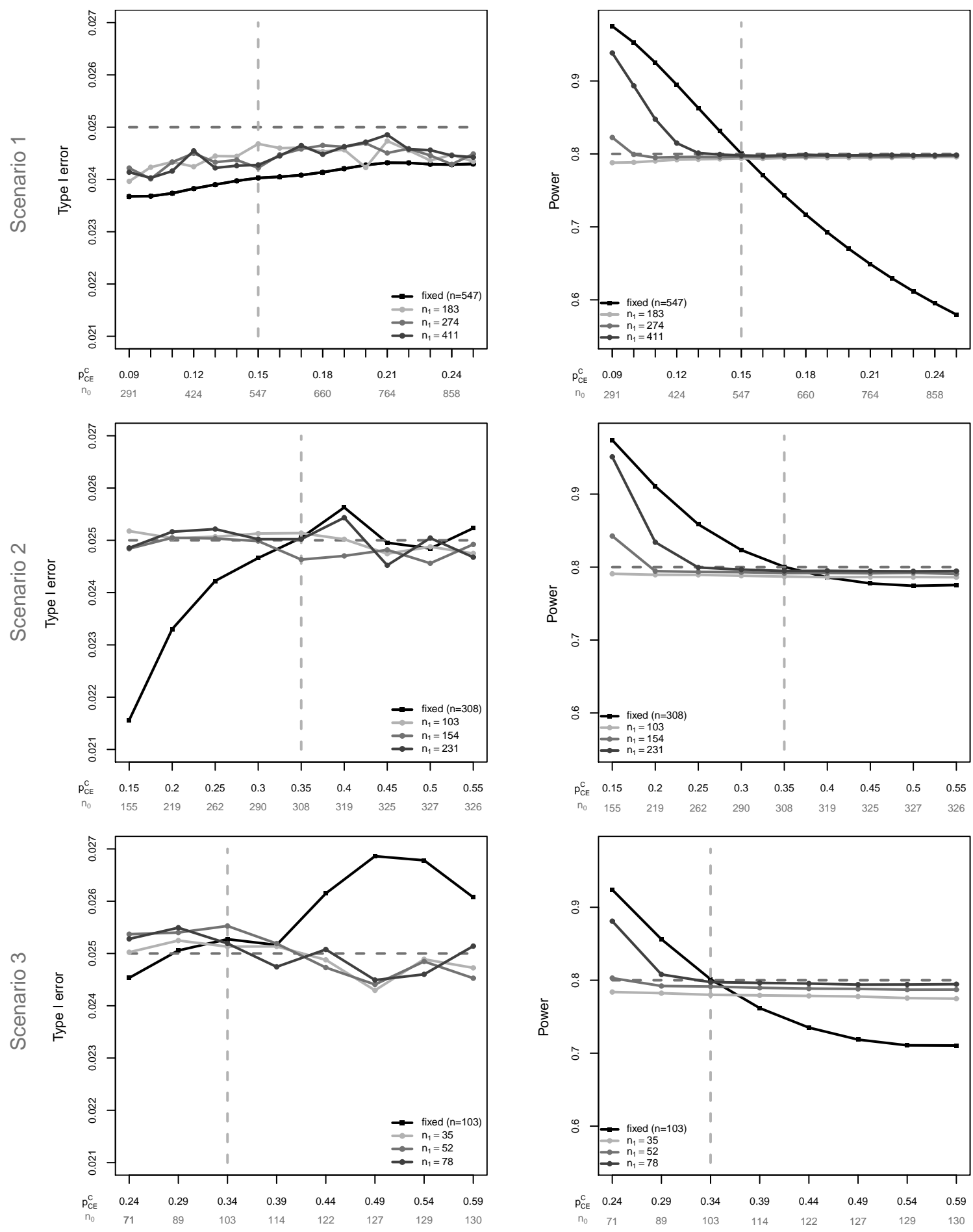

Figure 1: The calculated actual type I error rate (left panel) and power (right panel) for the fixed sample size design (black line) and simulated results for the internal pilot study design (grey lines). Results are shown for investigated scenarios 1 (top), 2 (middle), and 3 (bottom), applying three different sample sizes of internal pilot study using the unrestricted recalculation approach (as described in Section 5.1). Underlying parameters are as follows: $\Delta_{C E}=0.06, p_{M C}=0.0125$, and $\Delta_{M C}=0.003$ (scenario 1); $\Delta_{C E}=0.1, p_{M C}=0.075$, and $\Delta_{M C}=0.05$ (scenario 2); $\Delta_{C E}=0.18, p_{M C}=0.08$, and $\Delta_{M C}=0.06$ (scenario 3); with the initial assumption for $p_{C E}^{C}$ of $0.15,0.35$, and 0.34 , respectively. The vertical reference line indicates the setting matching the initial assumptions. Below the plots, true required sample sizes per group $n_{0}$ are given in grey color. 

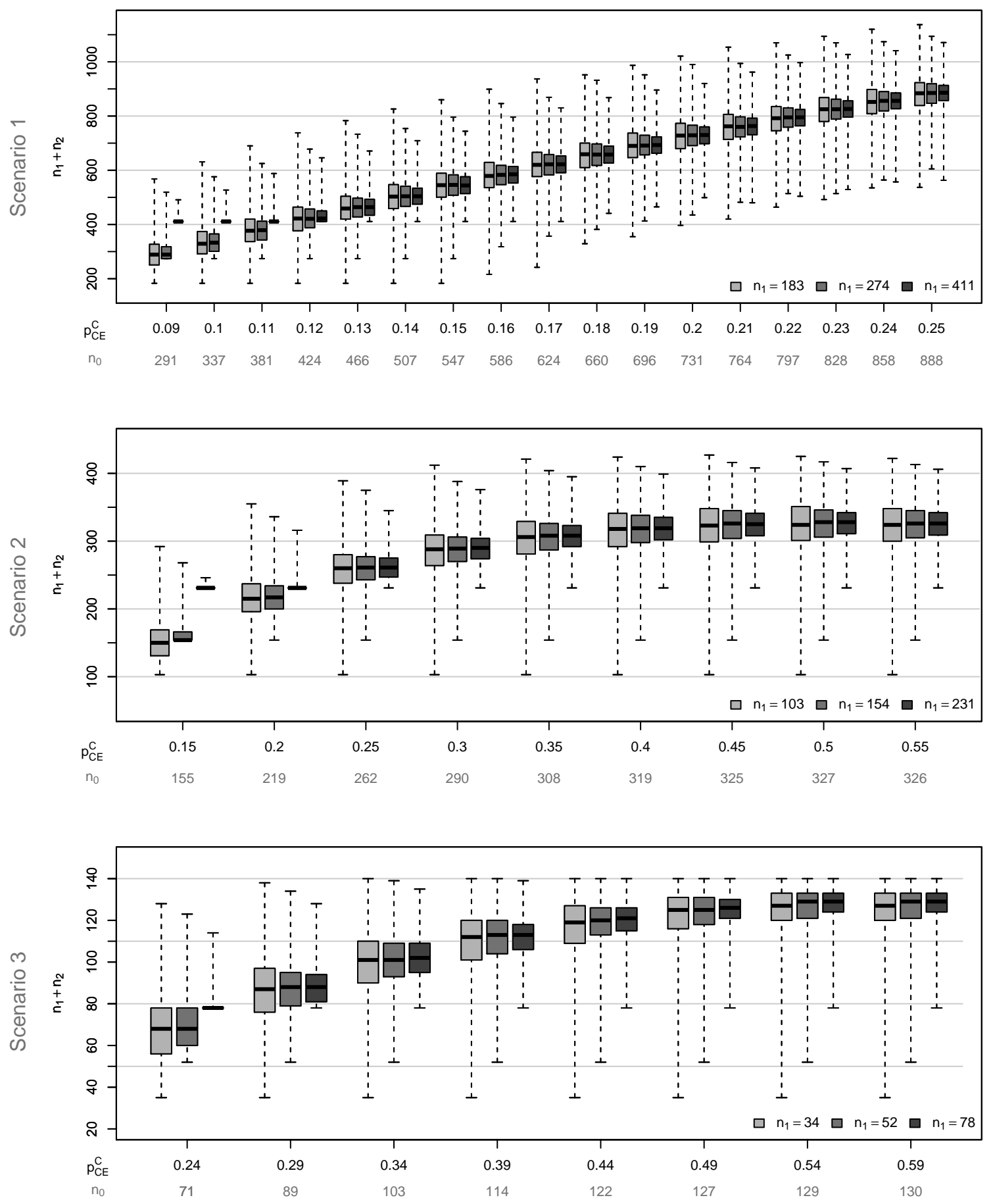

Figure 2: Simulated empirical distribution of the final sample size. The boxes show the median value and interquartile range of the final sample size $\left(n_{1}+n_{2}\right)$ and the whiskers indicate minimum and maximum. Results are shown for investigated scenarios 1 (top), 2 (middle), and 3 (bottom), applying three different sample sizes of internal pilot study using the unrestricted recalculation approach (as described in Section 5.1). Underlying parameters are as follows: $\Delta_{C E}=0.06, p_{M C}=0.0125$, and $\Delta_{M C}=0.003$ (scenario 1); $\Delta_{C E}=0.1, p_{M C}=0.075$, and $\Delta_{M C}=0.05$ (scenario 2); $\Delta_{C E}=0.18$, $p_{M C}=0.08$, and $\Delta_{M C}=0.06$ (scenario 3); with the initial assumption for $p_{C E}^{C}$ of $0.15,0.35$, and 0.34 , respectively. Below the plots, true required sample sizes per group $n_{0}$ are given in grey color. 